\title{
Implementación de prácticas de laboratorio en la educación virtual de los programas de ingeniería electrónica y telecomunicaciones
}

Elber Fernando Camelo-Quintero ${ }^{1}$

Universidad Nacional Abierta y a Distancia

elber.camelo@unad.edu.co

ORCID: https://orcid.org/0000-0002-9102-9655

DOI: https://doi.org/10.21158/2357514x.v7.n1.2019.2319

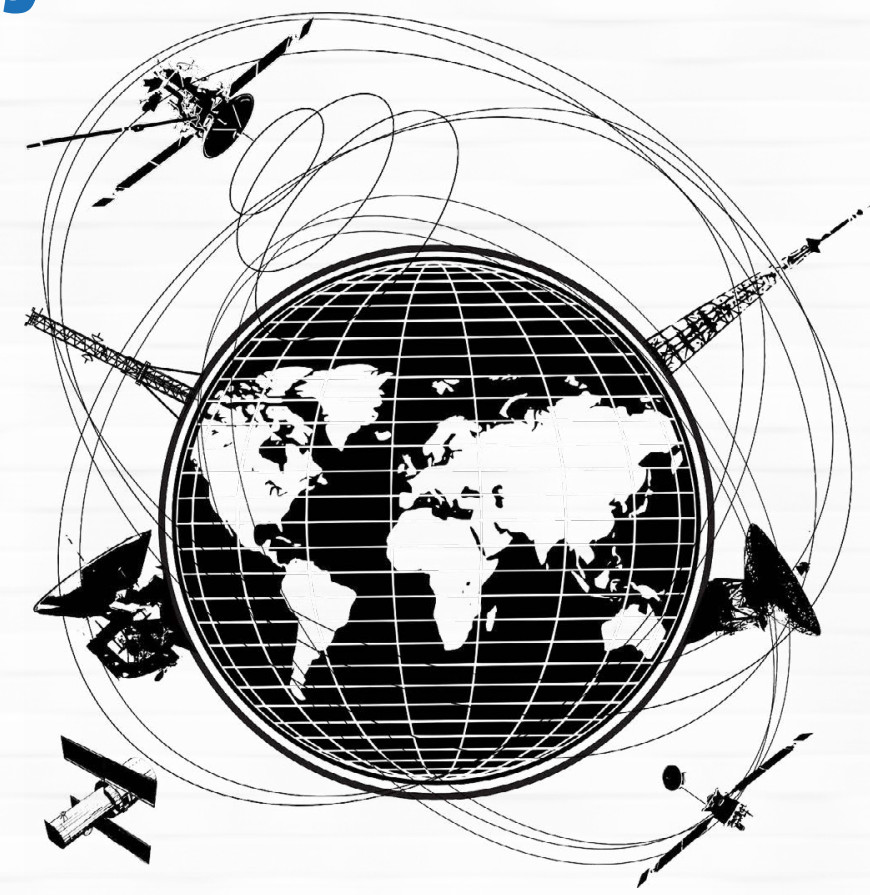

Cómo citar este artículo: Camelo-Quintero , E. F. (2019). Implementación de prácticas de laboratorio en la educación virtual de los programas de ingeniería electrónica y telecomunicaciones. Revista Virtu@lmente, 7(1), 29-44. DOI: https://doi.org/10.21158/2357514x.v7.n1.2019.2319

Fecha de recepción: 18 de enero de 2019

Fecha de aprobación: 21 de junio de 2019 


\section{RESUMEN}

Actualmente, la educación virtual permite a millones de personas formarse en diferentes áreas del conocimiento; por esta razón, la Universidad Nacional Abierta y a Distancia - UNAD ha respondido a esta realidad y oferta actualmente todos sus programas en la modalidad virtual.

La formación en programas de ingeniería ha suscitado interrogantes frente a la manera en que se deben realizar las prácticas de laboratorio, parte vital del proceso de enseñanza-aprendizaje del ingeniero; en esta investigación se realiza un estudio específico sobre los programas de Ingeniería Electrónica y de Telecomunicaciones, orientados en modalidad virtual y sobre la integración de las prácticas de laboratorio en un curso disciplinar específico y común a las dos ingenierías.

El presente artículo da a conocer las modalidades de laboratorio en los cursos metodológicos de la UNAD, una propuesta sobre cómo implementar las modalidades de laboratorio en la educación virtual y la importancia de articular las dos modalidades — presencial y simulada - con las competencias propuestas en un curso metodológico de los programas de Ingeniería Electrónica e Ingeniería de Telecomunicaciones.

A la luz de los resultados obtenidos, en los que el $87 \%$ de los docentes y el $79 \%$ de los estudiantes manifestaron que deberían implementarse las dos modalidades simultáneamente, se concluye que existe una complementariedad entre ellas. Esta conclusión se refuerza con el hecho de que los estudiantes que desarrollaron las dos modalidades de prácticas de laboratorio obtuvieron un desempeño satisfactorio en la prueba final donde se pusieron en juego las competencias del curso.

Palabras clave: educación virtual; educación a distancia; Ambientes Virtuales de Aprendizaje; enseñanza de ingeniería; simuladores; herramientas de enseñanza; proceso de enseñanza. 


\section{Implementation of laboratory practices in the virtual education of the Electronic Engineering and Telecommunications Engineering programs}

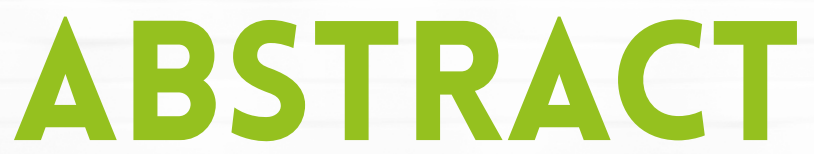

Virtual education currently allows millions of people to be trained in different areas of knowledge, for this reason, the Universidad Nacional Abierta y a Distancia - UNAD has responded to this reality and at the present time, it offers all its programs in the virtual mode.

Training in engineering programs has raised questions about the way in which laboratory practices, a vital part of the engineer's teaching-learning process, should be performed. In this investigation, we carried out a specific study on the programs of Electronic Engineering and Telecommunications Engineering, oriented in virtual modality and on the integration of the laboratory practices in a specific disciplinary course that is common to both engineerings.

This article presents laboratory modalities in UNAD methodological courses, a proposal on how to implement laboratory modalities in virtual education and the importance of articulating the two modalities (On-site and simulated), with the competences proposed in a methodological course of the Electronic Engineering and Telecommunications Engineering programs.

In the light of the results that were obtained, in which $87 \%$ of teachers and $79 \%$ of students stated that both modalities should be implemented simultaneously, it is concluded that there is a complementarity between them. This conclusion is reinforced by the fact that the students who developed the two modalities of laboratory practices, obtained a satisfactory performance in the final test where the competences of the course were put at stake.

Keywords: virtual education, long distance education, Virtual Learning Environments, engineering education, simulators, teaching tools, teaching process. 


\section{Implementação de práticas de laboratório no ensino virtual dos programas de Engenharia Eletrônica e Telecomunicações}

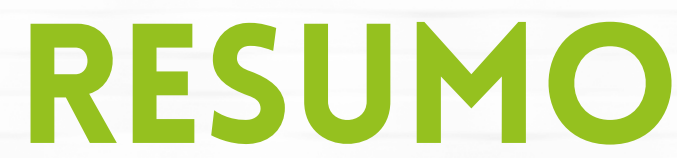

Atualmente, a educação virtual permite que milhões de pessoas sejam treinadas em diferentes áreas do conhecimento, por esse motivo, a Universidade Nacional Aberta e a Distância - UNAD respondeu a essa realidade e atualmente oferece todos os seus programas na modalidade virtual.

A formação em programas de engenharia levantou questões sobre como as práticas de laboratório devem ser realizadas, parte vital do processo de ensino-aprendizagem do engenheiro; nesta pesquisa é realizado um estudo específico sobre os programas de Engenharia Eletrônica e de Telecomunicações, orientados na modalidade virtual e sobre a integração das práticas de laboratório em um curso disciplinar específico e comum às duas áreas de engenharia.

Este artigo apresenta modalidades laboratoriais nos cursos metodológicos da UNAD, uma proposta sobre como implementar modalidades laboratoriais na educação virtual e a importância de articular as duas modalidades - presencial e simulada -, com as competências propostas em um curso metodológico dos programas de Engenharia Eletrônica e Engenharia de Telecomunicações.

À luz dos resultados obtidos, nos quais $87 \%$ dos professores e $79 \%$ dos alunos afirmaram que ambas as modalidades devem ser implementadas simultaneamente, conclui-se que existe uma complementaridade entre elas. Esta conclusão é reforçada pelo fato de os alunos que desenvolveram as duas modalidades de práticas laboratoriais terem obtido um desempenho satisfatório na prova final onde as competências do curso foram colocadas em jogo.

Palavras-chave: educação virtual; educação a distância; Ambientes Virtuais de Aprendizagem; ensino de engenharia; simuladores; ferramentas de ensino; processo de ensino. 


\section{Mise en place de pratiques de laboratoire pour l'enseignement virtuel des programmes d'ingénierie électronique et de télécommunications}

\section{RÉSUME}

L'éducation virtuelle permet de nos jours à des millions de personnes de se former dans une multitude de domaines de la connaissance. Pour cela, I'Université Nationale Ouverte et à Distance - UNAD - a embrassé cette réalité en offrant tous ses programmes en modalité virtuelle.

La formation aux programmes d'ingénierie a néanmoins soulevé des questions sur la manière dont les pratiques de laboratoire, étant un élément essentiel du processus d'enseignement-apprentissage de l'ingénieur, devaient être mises en œuvre. Une étude spécifique a été menée pour les programmes d'ingénierie électronique et de télécommunication en modalité virtuelle et sur l'intégration des pratiques de laboratoire de disciplines spécifiques et communes aux deux formations.

Cet article présentera les modalités de laboratoire des cours méthodologiques de I'UNAD, abordera la mise en place des modalités de laboratoire en éducation virtuelle, et l'importance de l'articulation des deux modalités - en salle de classe et à distance - et des compétences proposées dans un cours méthodologique pour les programmes mentionnés.

Selon les résultats de l'étude, $87 \%$ des enseignants et $79 \%$ des étudiants déclaraient que les deux modalités devaient être implémentées simultanément car fortement complémentaires. Cette conclusion est renforcée par le fait que les étudiants ayant mis au point les modalités de pratiques de laboratoire ont obtenu des résultats satisfaisants lors des examens finaux.

Mots-cléfs: éducation virtuelle; éducation à distance; environnements d'apprentissage virtuels; formation d'ingénieur; simulateurs; outils pédagogiques; processus d'enseignement. 


\section{Introducción}

Es indispensable reconocer la importancia de las prácticas de laboratorio en los programas de ingeniería, pues en estos espacios el estudiante puede poner a prueba los conceptos, leyes, conocimientos e ideas que surgen de la lectura de teoría, explicaciones de los docentes, inquietudes temáticas e ideas; esta importancia es evidente para Cruz y Valencia (2005), quienes mencionan la necesidad de potenciar el análisis y creatividad de los estudiantes de ingeniería mediante trabajos de laboratorio interactivos que privilegien el razonamiento sobre el trabajo repetitivo.

La Universidad Nacional Abierta y a Distancia (UNAD) es pionera en el modelo de enseñanza virtual basado en las múltiples herramientas que ofrecen las TIC; por esta razón, ofrece carreras tecnológicas, profesionales, especializaciones, maestrías y doctorados bajo la modalidad virtual.

En ingeniería, la práctica adquiere mayor relevancia, en comparación con otras áreas del conocimiento. En su quehacer diario, el ingeniero está en contacto permanente con la realidad y se enfrenta a diversas situaciones que pueden ser expresadas en un aula. Hace más de 20 años lo expresó Sánchez (1987), cuando afirmó que el ingeniero aprende «haciendo» en la vida real; lo que implica que, en su formación, el ingeniero debe dedicar mucho tiempo a la práctica si desea desarrollar competencias en el manejo de equipos y aplicar sus conocimientos en situaciones que le permitan el contacto permanente con la realidad. Por otro lado, Calvo, Zulueta, Gangoiti y López (2008) expresan que realizar experimentos con equipos reales es fundamental para el proceso de formación en disciplinas técnicas y científicas.

Tal es el caso de los programas académicos de Ingeniería Electrónica y de Telecomunicaciones, caracterizados generalmente por un altísimo componente práctico —manejo de equipos, insumos y procedimientos-, necesario para que el egresado de estos programas cumpla con las competencias mínimas establecidas por el mercado laboral y los diferentes programas de posgrado específicos sobre las diferentes líneas de la electrónica y las telecomunicaciones.

De este modo, la investigación se enfocó en determinar la manera de implementar la práctica de laboratorio, para que el estudiante pueda desarrollar las competencias propuestas para los cursos metodológicos en los programas de Ingeniería Electrónica e Ingeniería de Telecomunicaciones en la UNAD. Se estableció una metodología de investigación de enfoque mixto, en la cual se implementaron instrumentos cuantitativos y cualitativos que se aplicaron a una muestra de estudiantes y docentes de Ingeniería Electrónica e Ingeniería de Telecomunicaciones; así mismo, se realizó un análisis sobre el alcance de competencias por parte de los estudiantes en el curso específico de instrumentación, el cual se caracteriza por contener prácticas de laboratorio. 


\section{Prácticas de laboratorio y simuladores computacionales}

Las prácticas de laboratorio son experiencias que permiten validar conceptos teóricos mediante el uso de diferentes elementos y la realización de procedimientos. El laboratorio se define como el espacio físico donde el estudiante y/o sujeto de aprendizaje pone en práctica y comprueba diferentes conceptos teóricos a través de la experimentación.

Se debe resaltar la definición para las prácticas de laboratorio que ofrece la UNAD y que se denominan «Entorno de Aprendizaje Práctico», que se trata del contexto educativo ofrecido al estudiante para que, de manera guiada, aplique los conocimientos adquiridos durante el proceso formativo. De este modo, se fomenta en los estudiantes la proposición y la creatividad para aplicar teorías a situaciones problemáticas reales; es un «vínculo bidireccional en el que teoría y práctica se asimilan mutuamente» (UNAD, 2011, p. 58); así, se da un nuevo sentido y significado al conocimiento.

Los espacios de enseñanza-aprendizaje toman actualmente relevancia, particularmente, los laboratorios virtuales. Sanz y Martínez (2005) los definen de una manera muy clara al indicar que un laboratorio virtual comprende la simulación de la realidad mediante el uso de procesadores que codifican leyes y procesos, para así obtener respuestas a situaciones similares a las que se presentan en la vida real.
Es importante definir con claridad qué es la simulación para comprender cómo puede intervenir en el proceso enseñanza aprendizaje. Delval (2002) define los simuladores como modelos de situaciones o fenómenos donde, según el propósito, se consideran los aspectos importantes y se desprecian los que no tienen incidencia importante. Según la definición y una revisión de los simuladores actuales usados en la electrónica -si bien han avanzado de manera relevante- estos se caracterizan por dejar de lado aspectos que -en teoría- pueden parecer secundarios pero que, en la realidad, son aspectos que afectan directamente el funcionamiento de un circuito electrónico. Por este motivo, cuando se implementa físicamente un circuito simulado, puede que no se obtenga el mismo funcionamiento descrito por el simulador y se deban realizar ajustes.

\section{Contexto histórico de las prácticas de laboratorio en ingeniería}

En la ingeniería, la formación debe ofrecer espacios y recursos suficientes que posibiliten el desarrollo de destrezas y competencias para desempeñarse en un mundo real, con situaciones inadvertidas en cualquier momento. Juliao (2011) 
propone que la reflexión sobre la práctica sea fundamental para el buen desarrollo de las competencias requeridas en un área; en este sentido, Calvo et al. (2008) manifiestan que cuando se trata de disciplinas técnicas y científicas, es necesario llevar a cabo experimentos y prácticas con equipos reales, que permitan consolidar los conocimientos teóricos previamente adquiridos en las aulas o en capacitaciones.

No obstante, en la nueva era digital, estos métodos se han venido complementando con diferentes simuladores, lo que ha permitido un mayor aprovechamiento del tiempo en el laboratorio real, puesto que hoy día, la práctica requiere pasos esenciales como la observación, interpretación, análisis y devolución creativa (Juliao, 2011).

Para Escamilla (2013) la enseñanza de las prácticas en las ingenierías se ha visto aventajada por la virtualidad, es decir, que se ha obtenido un logro frente al mejoramiento de las competencias de los estudiantes, teniendo la virtualidad como complemento y no como remplazo, lo que se acerca al propósito de la investigación: descubrir las competencias adquiridas por lo estudiantes de las ingenierías.

En la era de la tecnología se observan grandes avances de manera continua y su implementación adquiere importancia en los procesos de formación; no obstante, aplicarla eficientemente conlleva una notoria relevancia si se habla en términos de calidad educativa. Algunos de esos avances tienen que ver con la identificación y definición de diferentes herramientas tecnológicas que se implementan para mejorar el proceso de enseñanza en dichas ingenierías.
Por otro lado, Lorandi, Hermida, Herández y Ladrón de Guevara (2011) hacen una comparación de la enseñanza en laboratorios a través de dos modalidades: de manera presencial y remota. Así, lograron identificar que la práctica presencial desarrolla algunas competencias profesionales en los estudiantes. Igualmente, cuando revisaron los resultados de la práctica remota, los estudiantes no solo realizaron el ejercicio de buena manera, sino también generaron competencias desde esta práctica. Concluyeron, entonces, que tanto las prácticas presenciales como las remotas en los laboratorios generan y desarrollan competencias profesionales que permiten la formación integral de los futuros ingenieros.

Se deduce así que el uso de laboratorios virtuales apoyados por software o simuladores computacionales ofrece a las diferentes instituciones múltiples beneficios en cuanto a optimización de recursos, cobertura y repetición indefinida de eventos hasta lograr el objetivo. En ese contexto, Guzmán, Torres y Bonilla (2014) sugieren diferentes metodologías para la enseñanza de la ingeniería, en las que ubican a los medios virtuales en la vanguardia de aquellos procesos; sin embargo, también concluyen que las prácticas presenciales no se pueden reemplazar en ninguna instancia por las virtuales.

Uno de los puntos más importantes de discusión sobre los simuladores computacionales estriba en si son realmente capaces de reemplazar a un laboratorio real y cómo lo podrían hacer, garantizando que en su proceso de uso se alcancen las mismas competencias. Al respecto Amaya (2008) concluye sus investigaciones diciendo que, en algunos casos, los simuladores computacionales, efectivamente, pueden reemplazar 
a un laboratorio real y que pueden permitir, además, aprender procedimientos. Sin embargo, se debe tener en cuenta que aquella investigación se refería a simuladores computacionales para el aprendizaje de la física, los cuales difieren de los simuladores usados en electrónica que, en muchas ocasiones, no tienen en cuenta variables externas al experimento que se desea realizar y lo afectan directamente.

Arias (2002) manifiesta que el uso de los simuladores computacionales puede constituirse en procedimiento, pero no en un método propiamente dicho; en su investigación concluyó que usar simulaciones desarrollaba habilidades en el uso de la computación y en el procesamiento de datos, dentro de contextos virtuales. Por otro lado, señaló que no se pueden considerar como un método, puesto que los simuladores necesitan de un complemento o procedimiento que pueda conectar la simulación con la realidad.

Luego de un estudio de seis años en la Universidad de Costa Rica, Monge y Méndez (2007) concluyeron que los simuladores superaron las expectativas y desplazaron de manera efectiva a las prácticas presenciales; sin embargo, se aclara que el área del conocimiento donde se realizó el estudio corresponde a las ciencias biológicas, las cuales difieren bastante de la electrónica y las telecomunicaciones, ingenierías de altísima exigencia en cuanto a competencias en el manejo de herramientas y dispositivos.

\section{La investigación}

\subsection{Técnicas e instrumentos}

Se enmarca en un sustento mixto; para su desarrollo, se utilizaron técnicas cualitativas y cuantitativas que permitieron fortalecer el proceso investigativo. La parte cualitativa consistió en la recopilación documental, la observación y las notas de campo; con el uso de las técnicas anteriormente descritas, se caracterizaron las prácticas de laboratorio en la UNAD, se analizó el desempeño del estudiante en el desarrollo de las prácticas de laboratorio y la manera como se relacionan estas con otras actividades del curso; así mismo, la interacción de aquel con sus compañeros y con el docente.

Cuantitativamente, se analizó la percepción de docentes y estudiantes acerca de las prácticas de laboratorio; para ello, se aplicó la escala Likert sobre las muestras de población, por separado, para estudiantes del curso instrumentación y de cursos metodológicos de los dos programas de ingeniería; con esto se buscó evitar el sesgo en las respuestas debido a las actividades propuestas en el curso. La segunda escala Likert se diseñó e implementó para los docentes de los diferentes cursos metodológicos de las ingenierías analizadas y tuvo como objetivo identificar la percepción que se tiene del componente práctico y la pertinencia de cada uno de los ambientes en los que este se desarrolla.

El análisis de la información incluyó un proceso de triangulación de la información, la cual permite conectar la información obtenida mediante diferentes instrumentos, pero de manera interactiva (Vallejo y de Franco, 2009). 
El ciclo de la investigación se compuso así:

Figura 1. Diseño de la investigación

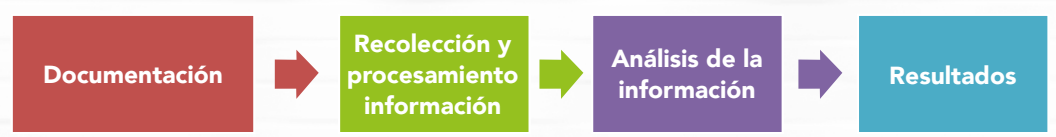

Fuente. Elaboración propia

\subsection{Actividades propuestas al estudiante}

Con el objeto de realizar el estudio comparativo sobre los dos tipos de práctica, se propusieron las actividades de la fase intermedia del curso en varias etapas: en un primer momento, los estudiantes realizan un desarrollo matemático básico para dar solución a un problema del área de instrumentación; esta parte se denomina «cálculos del diseño».

En un segundo momento, los estudiantes simulan su diseño mediante el uso de simuladores de circuitos electrónicos, tales como PSpice, Proteus, Circuitlab u otro. En un tercer momento, los estudiantes llevan el diseño al entorno real, tal como lo hicieron en la simulación; es en este paso donde se puede comprobar hasta qué punto los simuladores permiten que los estudiantes desarrollen las competencias que se plantean en el syllabus del curso.

En un cuarto momento, se solicita a los estudiantes realizar los ajustes necesarios - si los hubiere-, para que el circuito real funcione adecuadamente.
En el quinto momento, son los estudiantes quienes, mediante un análisis basado en ciertas preguntas detonadoras, dan a conocer la importancia de los dos tipos de prácticas de laboratorio desarrolladas.

En el sexto y último momento, los estudiantes analizan de manera retrospectiva el desarrollo total del curso, en particular, el del componente práctico, por ser el momento en el que habrán explorado los dos tipos de componentes prácticos que propone la UNAD; a esta instancia llegan guiados por unas preguntas detonadoras finales.

\section{Resultados}

\subsection{Caracterización de las modalidades de laboratorio}

La caracterización de las modalidades de laboratorio se realizó luego de una lectura detallada de los lineamientos de la UNAD, específicamente, sobre el área de laboratorios. A continuación, se presentan de manera concreta las características principales de la modalidad presencial y la modalidad basada en simulador, a la que se ha denominado modalidad simulada. 
Tabla 1. Caracterización de las modalidades de laboratorio

\begin{tabular}{|c|c|c|}
\hline Ítem & Modalidad presencial & Modalidad simulada \\
\hline Lugar & $\begin{array}{c}\text { El estudiante selecciona } \\
\text { la sede en la que desea } \\
\text { realizar los laboratorios. La } \\
\text { oferta de sedes la realiza } \\
\text { la UNAD. }\end{array}$ & $\begin{array}{c}\text { El estudiante realiza las } \\
\text { actividades desde el lugar } \\
\text { que desee. }\end{array}$ \\
\hline $\begin{array}{c}\text { Horas de } \\
\text { trabajo }\end{array}$ & $\begin{array}{l}\text { El estudiante debe dedicar } \\
\text { entre } 12 \text { y } 16 \text { horas de } \\
\text { trabajo en laboratorio, } \\
\text { dependiendo del número } \\
\text { de créditos del curso y de } \\
\text { las actividades a desarrollar. }\end{array}$ & $\begin{array}{c}\text { El estudiante debe dedicar } \\
\text { el tiempo que sea necesario } \\
\text { para el buen desarrollo de la } \\
\text { actividad. }\end{array}$ \\
\hline Equipos & $\begin{array}{c}\text { El estudiante encuentra } \\
\text { los equipos y } \\
\text { herramientas necesarias } \\
\text { para el desarrollo de } \\
\text { las actividades en el } \\
\text { laboratorio. El estudiante } \\
\text { debe llevar algunos } \\
\text { insumos específicos. }\end{array}$ & $\begin{array}{c}\text { El estudiante debe tener } \\
\text { nociones de manejo del } \\
\text { simulador. } \\
\text { Existen dos cursos dedicados } \\
\text { solo al manejo de los } \\
\text { diferentes simuladores } \\
\text { usados. }\end{array}$ \\
\hline $\begin{array}{c}\text { Desarrollo } \\
\text { práctica }\end{array}$ & $\begin{array}{l}\text { El estudiante debe realizar } \\
\text { en el tiempo disponible } \\
\text { (tres o cuatro horas por } \\
\text { encuentro), el desarrollo de } \\
\text { las actividades planteadas } \\
\text { en la guía de laboratorio. }\end{array}$ & $\begin{array}{l}\text { Se requiere un computador } \\
\text { con un simulador, de los } \\
\text { sugeridos por el docente } \\
\text { ( } 2 \text { simuladores sugeridos, al } \\
\text { menos } 1 \text { de versión libre). }\end{array}$ \\
\hline El docente & $\begin{array}{l}\text { Un docente diferente en } \\
\text { cada sede de práctica: } \\
\text { es guía y asesor para la } \\
\text { actividad, resuelve dudas } \\
\text { sobre las temáticas, } \\
\text { orienta sobre el manejo de } \\
\text { equipos, revisa y valora el } \\
\text { trabajo realizado por } \\
\text { los estudiantes. }\end{array}$ & $\begin{array}{l}\text { Un mismo docente que } \\
\text { acompaña al estudiante } \\
\text { en campus y tiene como } \\
\text { deber orientar y resolver } \\
\text { inquietudes sobre el } \\
\text { desarrollo de la temática. }\end{array}$ \\
\hline
\end{tabular}

Fuente. Elaboración propia.

\subsection{Análisis del desempeño de los estudiantes}

Al analizar el desempeño de los estudiantes en las prácticas presenciales como actividades del curso -incluyendo actividades con el simulador computacional-, se obtuvieron los siguientes resultados:

Se analiza el rendimiento académico de 35 estudiantes, quienes finalizaron el curso; con esto podemos eliminar distorsión en la estadística, por ejemplo, con estudiantes que, al obtener bajas calificaciones, desisten de continuar con el curso. Teniendo en cuenta las notas y el diario de campo, el análisis que se presenta a continuación es una correlación que tiene las siguientes variables:

a). Dependiente: la actividad final del curso.

b). Independiente: la práctica en modalidad simulada y la práctica en modalidad presencial.

En este orden de ideas, se mostrará un balance en el cual se identifican las formas en que varía la formación, según la práctica realizada por los estudiantes. 
Figura 2. Desempeño de los estudiantes en modalidad de laboratorio presencial vs. Desempeño en actividad final

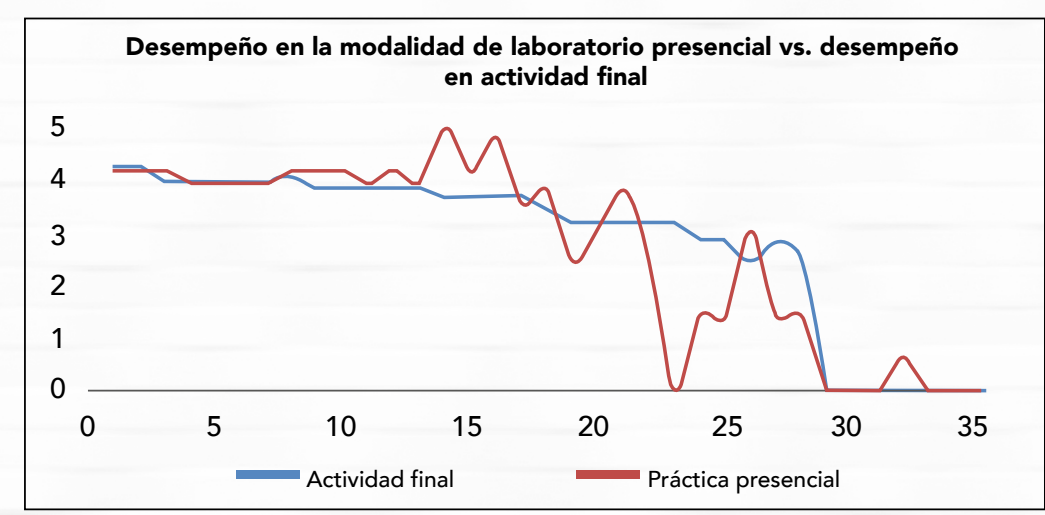

Fuente. Elaboración propia.

Figura 3. Desempeño de los estudiantes en modalidad de laboratorio simulado vs. Desempeño en actividad final

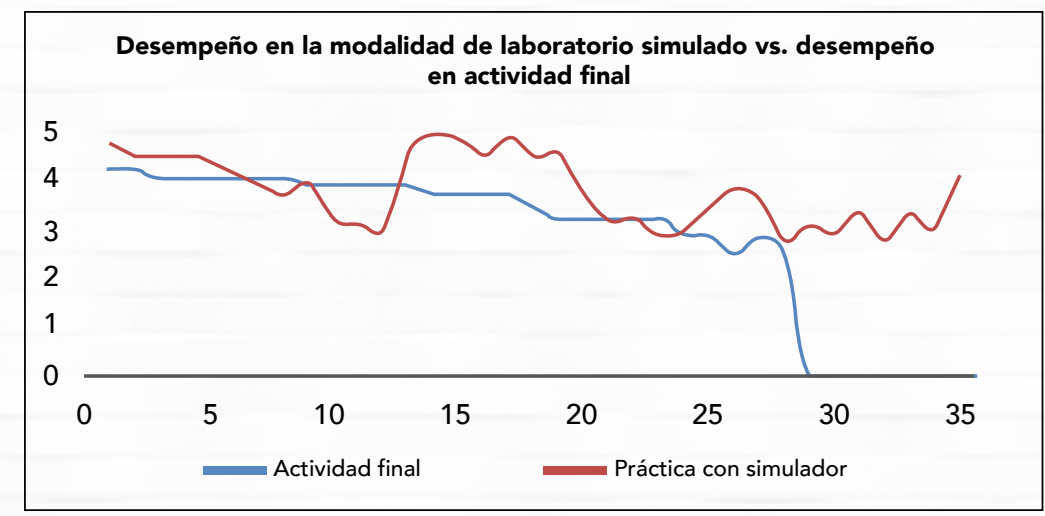

Fuente. Elaboración propia.

\subsection{La percepción de los estudiantes}

Se aplicó el instrumento cuantitativo, un cuestionario escala Likert a los estudiantes del curso de instrumentación, con respuesta oportuna de 27 estudiantes de 35 que realizaron actividades más allá de la mitad del curso; esto arroja un porcentaje de respuesta de $77,1 \%$ del total de estudiantes y un nivel de confianza del $93 \%$.

\subsection{Percepción de los docentes}

Se aplicó el instrumento cuantitativo cuestionario escala Likert a 31 docentes, de los 38 docentes de cursos disciplinares y laboratorio, en los programas de Ingeniería Electrónica e Ingeniería de Telecomunicaciones, con más de un (1) año de antigüedad en la UNAD, con el objeto de garantizar las modificaciones de los procesos y procedimientos que se han realizado en la UNAD sobre los componentes prácticos. Con los valores de población y muestra, se puede garantizar una certeza de $93 \%$, para el instrumento aplicado.

\section{Discusión}

Para empezar, se debe tener en cuenta que los diferentes instrumentos que se aplicaron fueron interpretados individualmente o, como se conoce en la investigación, análisis secuencial; de tal manera, la discusión se orienta a la convergencia de los resultados del trabajo investigativo.

La teoría de Ausubel (2002) plantea que las personas pueden almacenar gran cantidad de información; no obstante, esta información se retiene mediante la conceptualización de la información recibida y procesada en la memoria. Para 
evocar este tipo de información, se deben generar prácticas, es decir, mediante la praxis, se puede generar un aprendizaje mucho más duradero.

Lo anterior pone en evidencia que las prácticas virtuales o simuladas conllevan gran cantidad de hipótesis que deberían realizarse de manera práctica y presencial para abstraer la teoría; así lo arrojan, por lo menos, los resultados de la investigación. Este resultado es soportado por Juliao (2011), quien propone la praxeología; esta teoría plantea que los estudiantes no retienen información si no se hace una práctica y una respectiva reflexión frente a esa acción. Dicho en otras palabras, los estudiantes que realizan prácticas simuladas están en un porcentaje menor de adquirir las competencias que el curso de instrumentación sugiere. En ese sentido, la simulación se observaría como un complemento de dicho curso.

Por otra parte, Kaplún (2010) propone la comunicación digital como una forma ágil de generar más conocimiento; no obstante, se orienta hacia que los procesos simulados no pueden suplir en ningún momento todos los escenarios materiales. Esta investigación corrobora la proposición de Kaplún (2010), al obtener en los resultados que hay estudiantes con un excelente desempeño en la práctica simulada, pero notablemente inferior en la prueba final; igualmente, se encontraron casos donde sucede todo lo contrario.

Adicionalmente, el $45 \%$ de los estudiantes manifestaron inconvenientes al llevar a la realidad lo que habían realizado en el simulador, sumado a un $22 \%$ que respondió «ni de acuerdo ni en desacuerdo», lo cual deja abierta la posibilidad de que tuvieron dudas al hacer el proceso o sencillamente no lo hicieron.

Figura 4. Resultados de la cuarta pregunta del cuestionario tipo escala Likert estudiantes

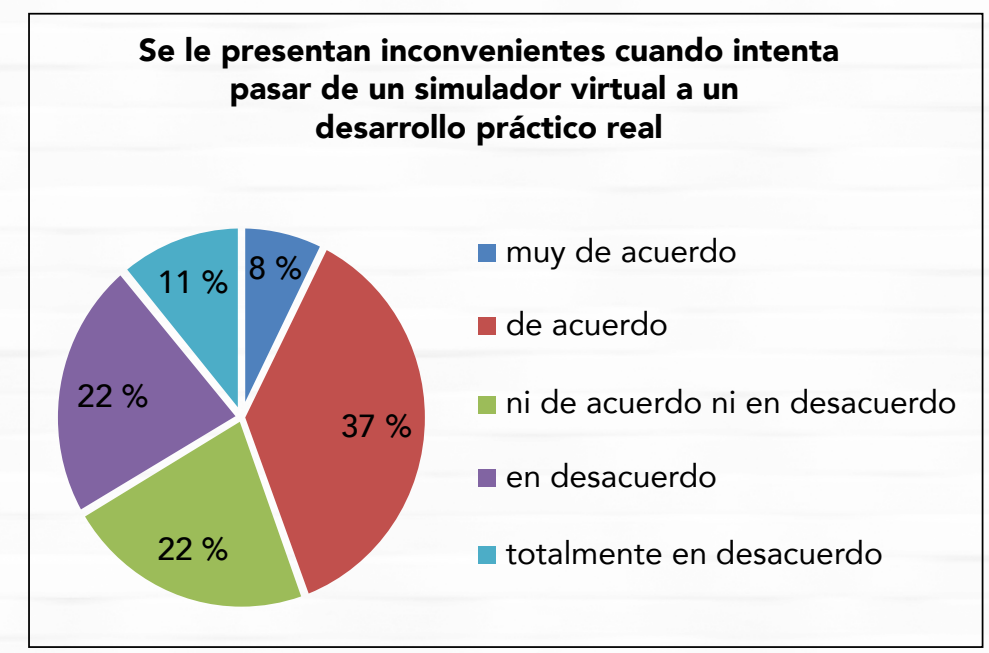

Fuente. Elaboración propia

Las competencias en los planes de trabajo de los docentes cumplen una función esencial, puesto que marcan la hoja de ruta a la hora de formar profesionales; la forma en que se midieron las competencias desarrolladas por los estudiantes del curso y su relación con las dos modalidades de prácticas de laboratorio permite inferir que es necesaria la modalidad presencial de laboratorio. Adicionalmente, los docentes son claros en dar su punto de vista al responder el cuestionario tipo escala Likert. A continuación, se dan a conocer las respuestas de dos de las preguntas.

Figura 5. Resultados de la sexta pregunta del cuestionario tipo escala Likert Docentes 


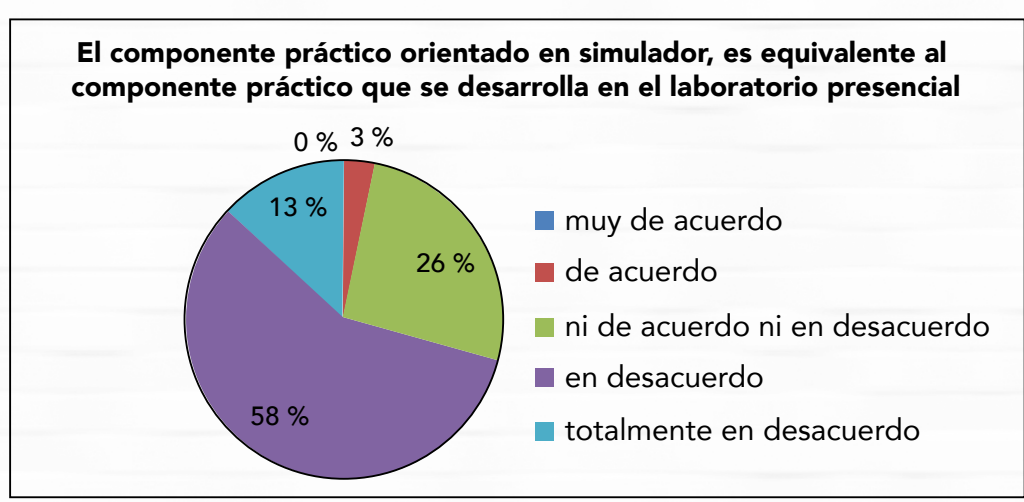

Fuente. Elaboración propia

Figura 6. Resultados de la séptima pregunta del cuestionario tipo escala Likert Docentes

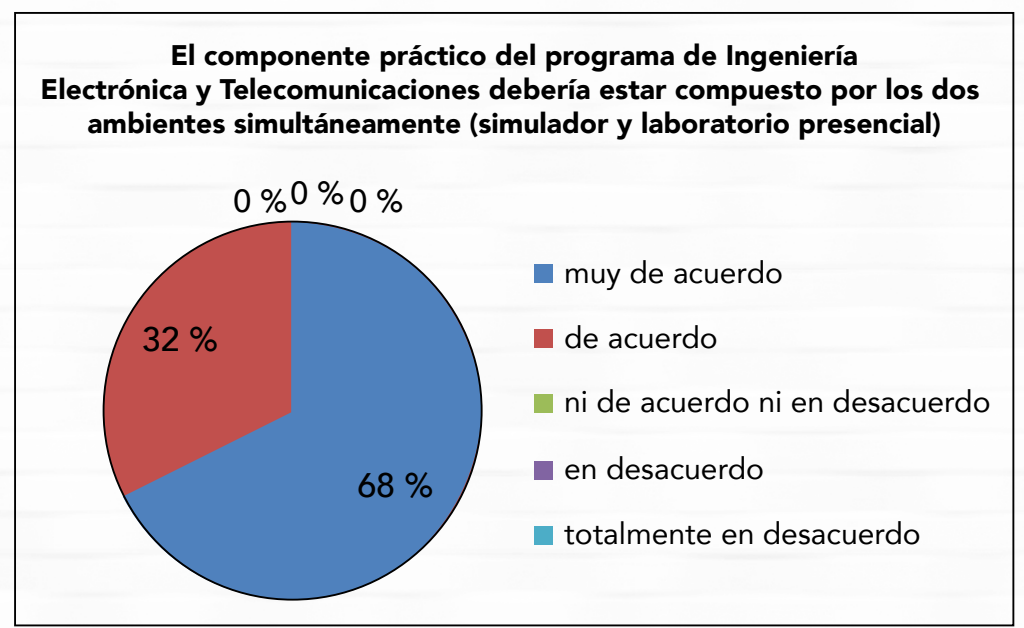

Fuente. Elaboración propia.

\section{Conclusiones}

Los cursos de instrumentación de los programas de Ingeniería Electrónica y Telecomunicaciones de la Universidad Nacional Abierta y a Distancia deben tener como principal componente las prácticas de laboratorio presenciales, teniendo como herramienta complementaria los simuladores computacionales, puesto que Vásquez y Gabalán (2006) plantean que, para el buen rendimiento profesoral, el docente no solo debe apoyarse en los constructos teóricos, sino que, por el contrario, es necesaria la práctica para refinar los saberes conceptuales sobre un ciencia específica, como en este caso de las ingenierías relacionadas para el estudio.

Lo anterior se corrobora con los resultados obtenidos en la investigación, en los que el $100 \%$ de los docentes reconoce el desarrollo de las prácticas de laboratorio como indispensables en la formación en ingeniería electrónica e ingeniería de telecomunicaciones, a la vez que indican que esta práctica de laboratorio debe estar compuesta por ambas modalidades.

Así mismo, Calvo et al. (2008) concuerdan con que el uso de laboratorios reales y virtuales — simulados - generan una complementariedad, que se puede evidenciar en un mayor aprovechamiento de las horas in situ del laboratorio, espacio donde los estudiantes se centran en resolver actividades es- 
pecíficas. A la luz de los resultados obtenidos, en los que el $87 \%$ de los docentes y el $79 \%$ de los estudiantes manifestaron que deberían implementarse las dos modalidades simultáneamente, se concluye que existe una complementariedad entre las mismas. Esta conclusión se refuerza con el hecho de que los estudiantes que desarrollaron las dos modalidades de prácticas de laboratorio, obtuvieron un desempeño satisfactorio en la prueba final donde se pusieron en juego, las competencias del curso.

Para los estudiantes, las prácticas presenciales son fundamentales y los simuladores requieren de una instrucción para que puedan ser manejados de manera adecuada. Esto aconseja tener un acompañamiento tutorial adecuado, con las horas necesarias para el buen manejo de las plataformas, igual que de la práctica presencial en laboratorio.

La modalidad de laboratorio simulada que se propone en el curso de instrumentación actualmente no es equivalente a la modalidad presencial; los simuladores usados desprecian fenómenos o situaciones críticas que pueden generar un pobre desarrollo de las competencias de los estudiantes. En lo anterior concuerdan tanto docentes como estudiantes de los programas de Ingeniería Electrónica e Ingeniería de Telecomunicaciones.

Si una de las razones para promover la implementación de la modalidad simulada sobre la modalidad presencial en las áreas de electrónica y telecomunicaciones estriba en el ahorro de dinero en espacios, insumos y manejo del tiempo por parte de docentes y estudiantes, ya se demostró su no equivalencia en esta investigación; se hace necesario entonces que se revise de manera detenida la opción de los laboratorios remotos que presentan las ventajas expuestas, sumado a que el estudiante puede observar y manipular remotamente fenómenos y dispositivos en la vida real; además, estos laboratorios pueden tener una disponibilidad de 24 horas al día.

\section{Referencias}

Amaya, G. (2008). La simulación computarizada como instrumento del método en el proceso de enseñanza y aprendizaje de la física. Revista Electrónica Actualidades Investigativas, 8(1), $1-31$.

Arias, L. (2002). La simulación computarizada como procedimiento metodológico en la enseñanza y el aprendizaje de la electrónica. Recuperado de http://www.ilustrados.com/ tema/3575/simulacion-computarizada-proceso-ensenanzaaprendizaje-Electronica.html

Ausubel, D. (2002). Adquisición y retención del conocimiento. una perspectiva cognitiva. Barcelona: Grupo Planeta.

Calvo, I.; Zulueta, E.; Gangoiti, U.; López, J. (2008). Laboratorios remotos y virtuales en enseñanzas técnicas y científicas. Ikastorratza, e-Revista de didáctica, 3, 1-21. 
Cruz, J. C.; Valencia, J. F. (2005). La formación práctica del ingeniero electrónico en el laboratorio. Revista Científica Guillermo de Ockham, 3(1), 115-130.

Delval, J. (2002). El desarrollo humano. Madrid: Siglo XXI editores.

Escamilla, J. G. (2013). Avances en la enseñanza a distancia de la Ingeniería. Revista de Ingeniería, 39, 67-72.

Guzmán, J.; Torres, I.; Bonilla, M. (2014). Un caso práctico de aplicación de una metodología para laboratorios virtuales. Scientia et technica, 19(1), 67-76.

Juliao, C. G. (2011). El enfoque praxeológico. Bogotá: Corporación Universitaria Minuto de Dios.

Kaplún, M. (2010). Una pedagogía de la comunicación. Madrid: Ediciones de la Torre.

Lorandi, A.; Hermida, G.; \& Herández, J.; Ladrón de Guevara, E. (2011). Los laboratorios virtuales y laboratorios remotos en la enseñanza de la ingeniería. Revista Internacional de Educación en Ingeniería, 4, 24-30.

Monge, J.; Méndez, V. H.(2007). Ventajas y desventajas de usar laboratorios virtuales en educación a distancia: la opinión del estudiantado en seis años de duración. Educación, 31 (1), 91 108. DOI: https://doi.org/10.15517/revedu.v31i1.1255

Sanz, A.; Martínez, J. L. (2005). El uso de los laboratorios virtuales en la asignatura Bioquímica como alternativa para la aplicación de las tecnologías de la información y la comunicación. Tecnología Química, 25(1), 5-17.
Sánchez, J. (1987). Elementos propios de la profesión en los currículos de ingeniería. En I. C. Superior, Planteamientos y reflexiones alrededor del currículo en la educación superior: Ciclo de conferencias (págs. 233-251).

Vallejo, R.; de Franco, M. (2009). La triangulación como procedimiento de análisis para investigaciones educativas. REDHECS, 4(7), 117-133.

Vásquez, F.; Gabalán, J. (2006). Percepciones estudiantiles y su influencia en la evaluación del profesorado. Relieve, 12(2), 219-245. 